\title{
Pneumatic Soft Actuator using Twist of Flexible H-Structure
}

\author{
Toshiro NORITSUGU* and Norio SUYAMA* \\ ${ }^{*}$ Department of Systems Engineering, Faculty of Engineering \\ Okayama University \\ 3-1-1 Tsushimanaka, Okayama, 700-8530 Japan \\ E-mail:toshiro@sys.okayama-u.ac.jp \\ norio@mcrlab.sys.okayama-u.ac.jp
}

\begin{abstract}
A new pneumatic soft actuator has been developed. The actuator is made of silicon rubber tubes, which is constructed in the H-structure. The actuator has three degrees of freedom, expanding in the vertical direction, bending in the horizontal direction and rotating around the vertical centerline of the $\mathrm{H}$-structure, each motion can be obtaind by adjusting the inner pressure of three silicon tube segments constructing the H-structure. This paper describes the principle of the actuator and some experimental results and discussion. The results shows the availability of the proposed actuator.
\end{abstract}

\section{KEY WORDS}

Pneumatic actuator, Soft actuator, Silicon rubber, Pressure control, Human friendly robotics,

\section{INTRODUCTION}

A human friendly robotics and mechatronics have attracted interest recently, which require a flexible mechanism. In order to assure the safety for human, such mechanism should be driven with an inherently soft actuator. The soft actuator has to be provided with both the flexibility for the environmental objects and the satisfactory controllability to attain the desired operation. A pneumatic actuation can become one of the most effective ways to satisfy the above requirements. A combination of pneumatic actuator with a proper control strategy is most available to realize the soft actuation system $[1],[2]$.

In this study, a new pneumatic soft actuator has been developed. The newly developed actuator is made of silicon rubber tubes, which are constructed in the H-structure. The actuator has three degrees of freedom, expanding in the vertical direction, bending in the horizontal direction and rotating around the vertical centerline of the H-structure, each mo- tion can be obtained by adjusting the inner pressure of three silicon tube segments constructing the $\mathrm{H}$ structure.

By coupling some $\mathrm{H}$-structure, an actuator with multi-degrees of freedom can be built up. Also some kinds of constructions of this actuator unit can be considered.

This paper describes the operational principle and the fundamental characteristics of this actuator unit.

\section{STRUCTURE OF ACTUATOR}

Figure 1 shows the principal structure of the actuator. It constructs a $\mathrm{H}$-structure with three silicon rubber tubes 1, 2 and 3. A tube 4 is an auxiliary one to deliver the output from the actuator. The tubes are bonded each other, but there is no air flow between each tube. The inner pressures in tubes from 1 to 3 are adjusted to make a desired configuration of the H-structure.

The tubes are reinforced with fibers as shown in Figure 2. Tube 1 and 2 are reinforced in the radial 


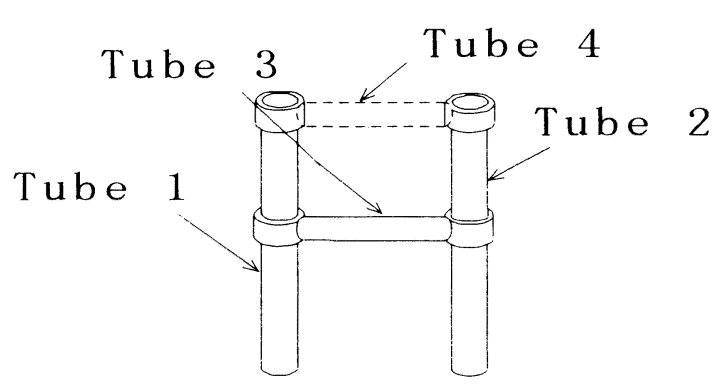

Figure 1: Structure of actuator

direction, so that, pressurizing these tubes, which stretch in the longitudinal direction rather than the radial direction. When reducing the pressure, the tubes return to the original length due to the own elasticity.

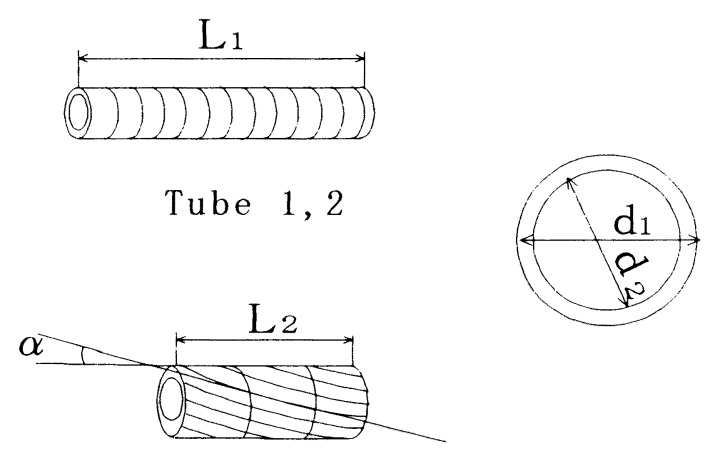

Tube 3

Figure 2: Reinforced tubes

The tube 3 is wrapped with a fiber around the circumference and in the longitudinal direction with an inclination angle of $\alpha$. Pressurizing the tube 3, it is twisted in the direction for the angle $\alpha$ to become zero. The maximum twist angle $\theta_{\max }$ depends on the tube size and the inclination angle, given by Eq.(1) as shown in Figure 3(a).

$$
\theta_{\max }=L_{2} \tan \alpha / \pi d_{1} \times 360
$$

Where it is assumed that the fiber does not expand and contract. $L_{2}, d_{1}$ and $\alpha$ are tube length, outer diameter of tube and inclination angle of fiber, respectively, in the initial condition. The tube length $L^{\prime}$ at the maximum twist angle is given by

$$
L^{\prime}=L_{2} / \cos \alpha
$$

Also, as shown in Figure 3(b). The relation between the changes of twist angle and tube length can be expressed by

$$
\begin{gathered}
\Delta \theta=\frac{L_{2} \tan \alpha-L_{2} \tan \alpha^{\prime}}{\pi d_{1}} \times 360 \\
\alpha^{\prime}=\cos ^{-1} \frac{L_{2}+\Delta L}{L^{\prime}}
\end{gathered}
$$

$\Delta \theta$ and $\Delta L$ denote the respective changes.

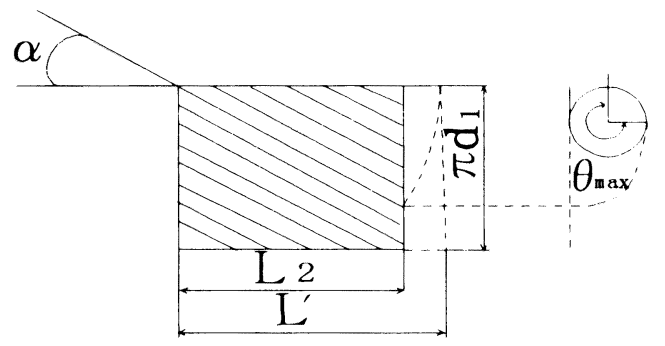

(a) Maximam twist angle

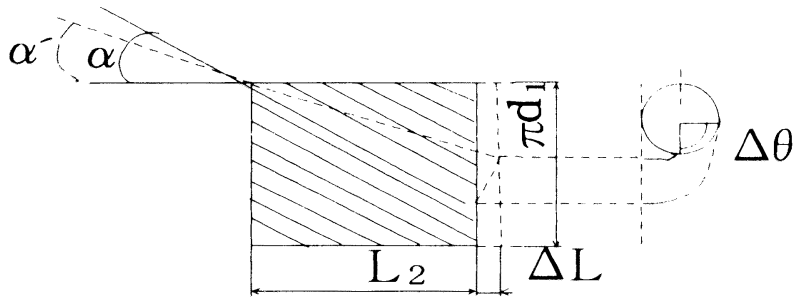

(b) Change of twist angle

Figure 3: Twist motion of tube 3

Figure 4(a) shows $\theta_{\max }$ calculated from Eq.(1). Figure $4(\mathrm{~b})$ shows the relation between $\Delta \theta$ and $\Delta L$ calculated from Eqs.(2),(3) and (4) for some values of $\alpha$. Where the initial tube length $L_{2}$ is assumed to be $60[\mathrm{~mm}]$, and the outer diameter of tube $d_{1}$ is $10[\mathrm{~mm}]$.

The large $\alpha$ increases $\theta_{\text {max }}$, but also the ratio of $\Delta L / \Delta \theta$ becomes large. It is not desired for the tube 3 to largely expand in the actuator of $\mathrm{H}$-structure when it twists. Therefore the value of inclination angle $\alpha$ should be as small as possible in the region where the desired $\theta_{\max }$ can be obtained.

\section{PRINCIPLE OF ACTUATOR}

Figure 5 shows the fundamental three motions of the actuator, where the bottoms of the tube 1 and 2 are assumed to be fixed. 


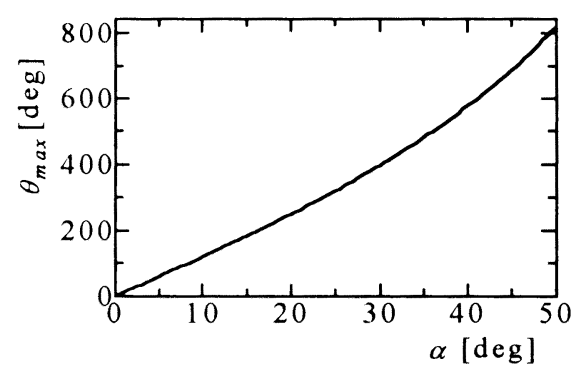

(a) Caluculated maximum twist angle

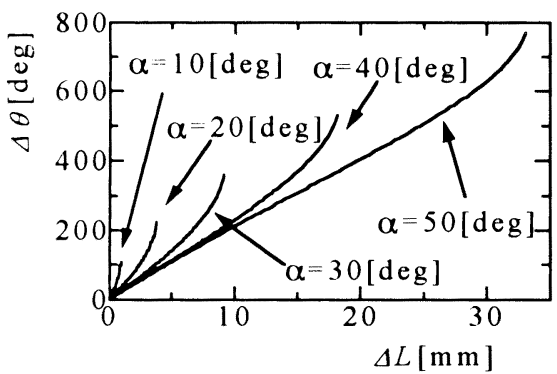

(b) Caluculated relation between $\Delta \theta$ and $\Delta L$

Figure 4: Fundamental property of tube 3

Figure 5(a) shows the expansion and contraction motion in the direction of $z$ axis. By uniformly increasing the pressures $P_{1}$ and $P_{2}$, respectively in the tube 1 and 2 , that is, $P_{1}=P_{2}$, the actuator expands, and by reducing them it contracts due to the elasticity of a silicon rubber. Figure $5(\mathrm{~b})$ shows the bending motion in the $y$ direction. By adjusting $P_{1}>P_{2}$, the actuator can bend to the direction of positive $y$ axis owing to the difference of the length of two tubes. When $P_{1}<P_{2}$, it can bend to the negative one. Figure $5(c)$ shows the twist motion around the $\approx$ axis, which is caused by pressuring the tube 3 .

As mentioned above, by adjusting $P_{1}, P_{2}$ and $P_{3}$ the actuator can attain the three degrees of freedom motion, of which the variable displacements are $x_{L}, \psi$ and $\theta_{2}$.

\section{EXPERIMENTAL SETUP}

Figure 6 shows the experimental setup. A silicon rubber tube with outer diameter of $10[\mathrm{~mm}]$ and wall thickness of $2[\mathrm{~mm}]$ is used. The tube segments are bonded each other by the silicon rubber adhesive. Photograph 1 shows an experimental actuator. The compressed air is supplied to each tube through a plastic connector from a pneumatic servo control valve. The inner pressures $P_{1}, P_{2}$ and $P_{3}$ are adjusted by the feedback controller using a personal

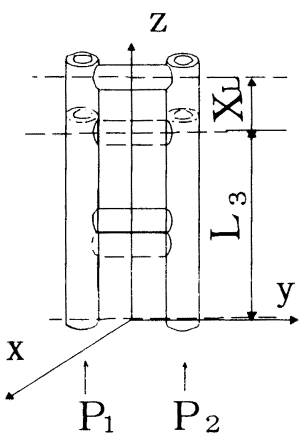

(a) Expansion and contraction motion

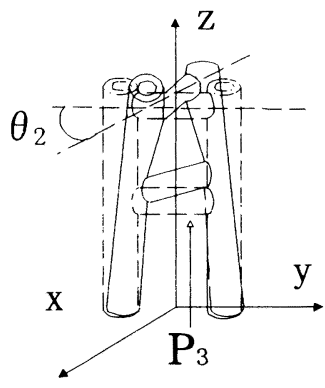

(c) Twist motion

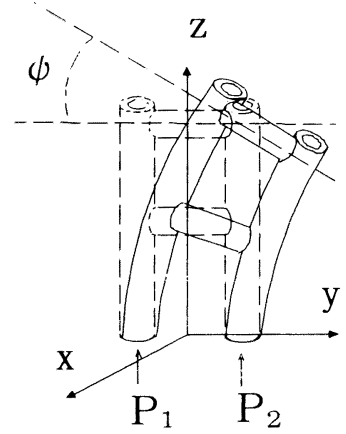

(b) Bending motion

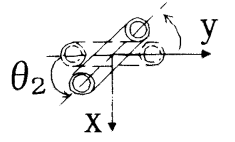

Figure 5: Fundamental three motions of actuator

computer. The variable displacements $x_{L}, \psi$ and $\theta_{2}$ are measured with a visual motion analyzer.

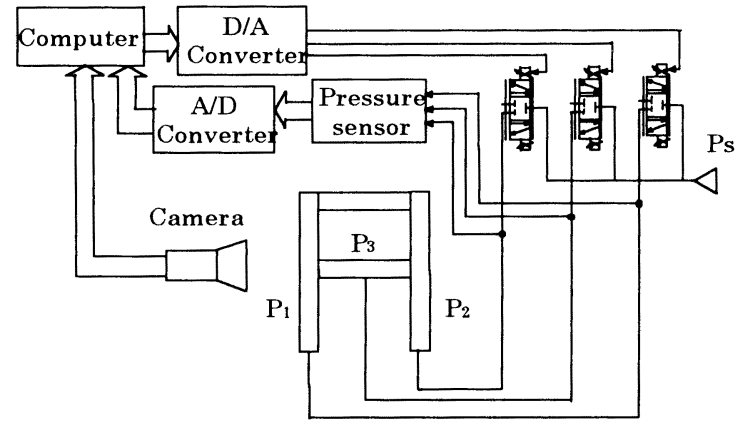

Figure 6: Experimental setup

\section{EXPERIMENTAL RESULTS AND DISCUSSION}

An experimental actuator is composed of the tubes 1 and 2 with length of $100[\mathrm{~mm}]$, outer diameter of $10[\mathrm{~mm}]$ and wall thickness of $1[\mathrm{~mm}]$, which are wrapped with a fiber every distance of $5[\mathrm{~mm}]$. The tube 3 has the length of $60[\mathrm{~mm}]$, the same diameter and thickness as tubes 1 and 2, which is wrapped at every distance of $15[\mathrm{~mm}]$ in the circumferential direction 


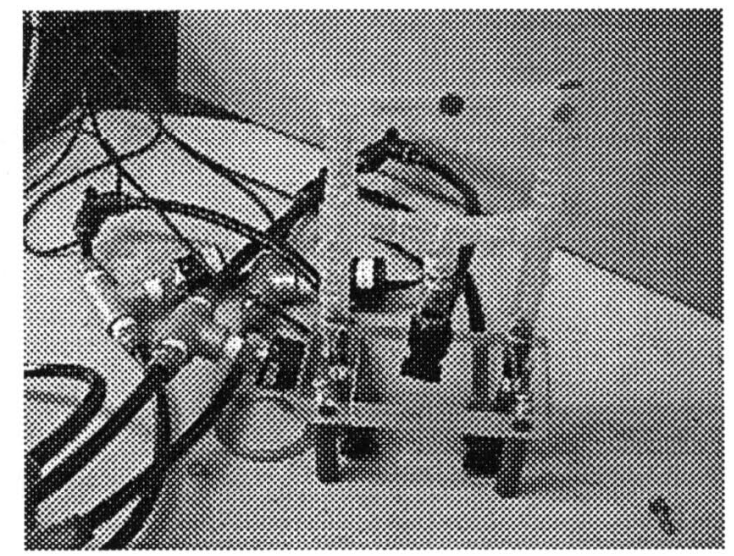

Photograph 1: Experimental actuator

and with the inclination angle $\alpha$ of $20[\mathrm{deg}]$ in the longitudinal direction.

\section{FUNDAMENTAL CHARACTERISTICS OF TUBES}

Figure 7(a) shows the expanding displacements of tube 1 and 2 for the inner pressure. A symbol of - denotes the average value, a symbol of vertical segment denotes the amount of scatter of measured values. For example, when the inner pressure $P=200 \mathrm{kPa}$, the tube 1 and 2 can expand by about $18 \%$.

The measured twist angle $\theta_{1}$ of the tube 3 is shown in Figure 7(b) for some values of inner pressure. When $P=200 \mathrm{kPa}$, the tube can twist about 150[deg].These longitudinal displacement and twist angle are expected to be sufficient to drive the $\mathrm{H}$ structure actuator.

\section{FUNDAMENTAL PERFORMANCE OF ACTUATOR}

A H-structure actuator is constructed by using tube 1,2,3 and 4 . The tube 3 and 4 are bonded at a height of $50[\mathrm{~mm}]$ and the top of tubes 2 and 3,respectively. The tube 4 is not pressurized, of which inner pressure is always atmospheric. The tube 1 and 2 build at a distance of $70[\mathrm{~mm}]$ between central axes each other.

Figure 8 (a) shows the expanding displacement $x_{L}$ of the actuator for some inner pressures $\left(P_{1}=P_{2}\right)$, which are measured at the center of the tube 4 . The expanding displacement is made a little smaller compared with the values for single tube shown in Figure 7 (a). The bonding parts between tube 1, 2 and 3 may cause this decrement.

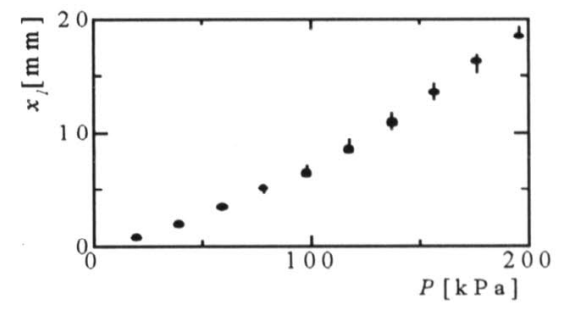

(a) Expanding displacements of tube 1 and 2

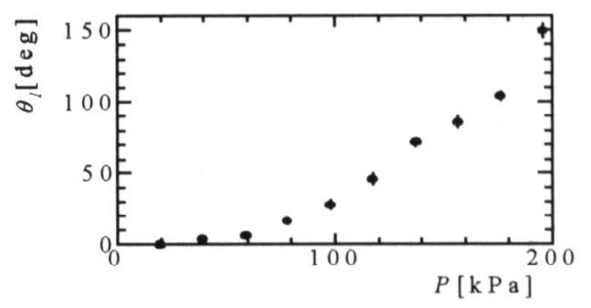

(b) Tw ist angle of tube 3

Figure 7: Fundamental characteristics of tubes



(a) Expanding displacement

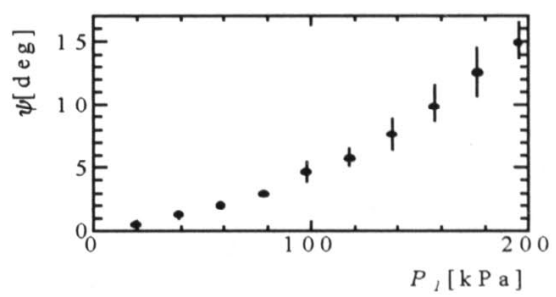

(b) Bending angle

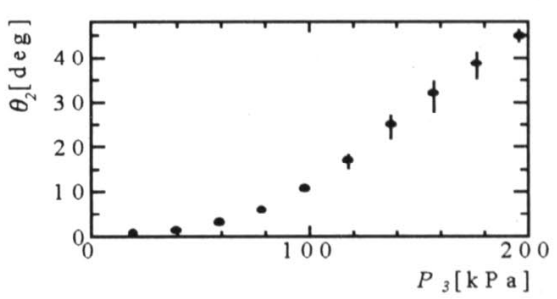

(c) T w ist angle

Figure 8: Fundamental performance of actuator 
Figure $8(\mathrm{~b})$ shows the bending angle $\psi$ to the direction of the $y$ axis at increasing $P_{1}$ when $P_{2}=$ 0 . Figure $8(\mathrm{c})$ shows the twist angle around the $z$ axis when only the tube 3 is pressurized, the other tubes are in the atmospheric pressure. There is a little hysteresis, but, since it is almost included in the range of the scatter, it is not illustrated in the figures.

The results shown in Figure 8 can prove the availability of the proposed actuator.

\section{MULTIPLE OPERATION}

Two degrees of freedom in the actuator are simultaneously driven to show the possibility of the multiple operation of the proposed actuator.

Figure 9 shows the combination of the displacement in the $z$ axis and the bending to the $y$ axis. It shows $x_{L}$ and $\psi$, respectively, measured at increasing the pressure $P_{1}$ in tube 1 for some values of the pressure $P_{2}$ in tube 2 shown in figures and $P_{3}=0 . \psi$ can be controlled by adjusting the pressure difference $P_{1}-P_{2}$. Since the tube 4 is displaced by bending, $x_{L}$ changes due to $P_{2}$.
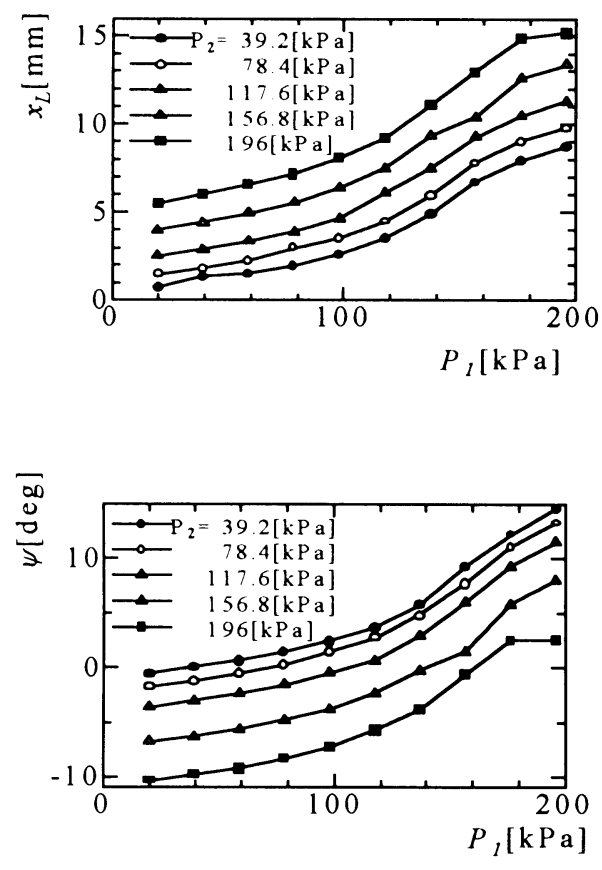

Figure 9: Combination of displacement $x_{L}$ and bending angle $\psi\left(P_{3}=0\right)$

Figure 10 shows the combination of the displacement $x_{L}$ in the $z$ axis and the twist angle $\theta_{2}$ around the $z$ axis. Such multiple operation can be realized by pressurizing both tube 1 and 2 at the same values and the pressure $P_{3}$ in tube 3 at some values shown in figures. When $\theta_{2}$ increases, $x_{L}$ is made decreased because the tube 1 and 2 are bent in the opposite directions each other by their twisting as shown in Figure 5 .
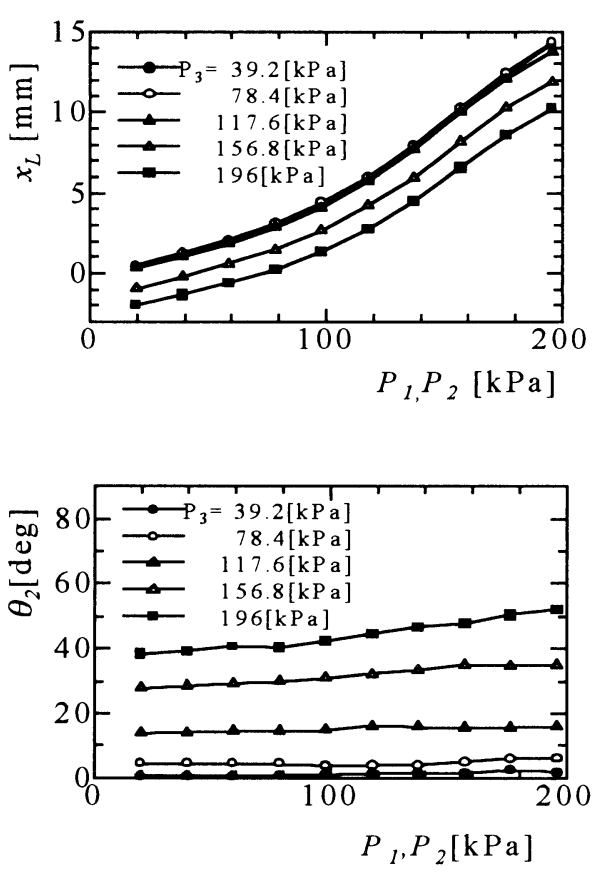

Figure 10: Combination of displacement $x_{L}$ and twist angle $\theta_{2}$

Figure 11 shows the combination of $\theta_{2}$ and $\psi$, which can be realized by pressurizing $P_{1}$ and $P_{3}$, when $P_{2}=0$. Both angles are shown in figures in which the values of $P_{3}$ are shown. Ideally $\psi$ is desired not to be influenced by the pressure $P_{3}$, however, it depends on also $P_{3}$.

The results can prove the possibility of multiple operation of the proposed actuator. However, a certain operational interaction exits due to the structure of actuator.

\section{CONCLUSION}

A pneumatic soft actuator made of silicon rubber tubes has been developed, which is based on a $\mathrm{H}$-structure and can operate with a three-degrees of freedom. This paper describes the principle of the actuator and some experimental results and discussion. The results have shown the availability of the 

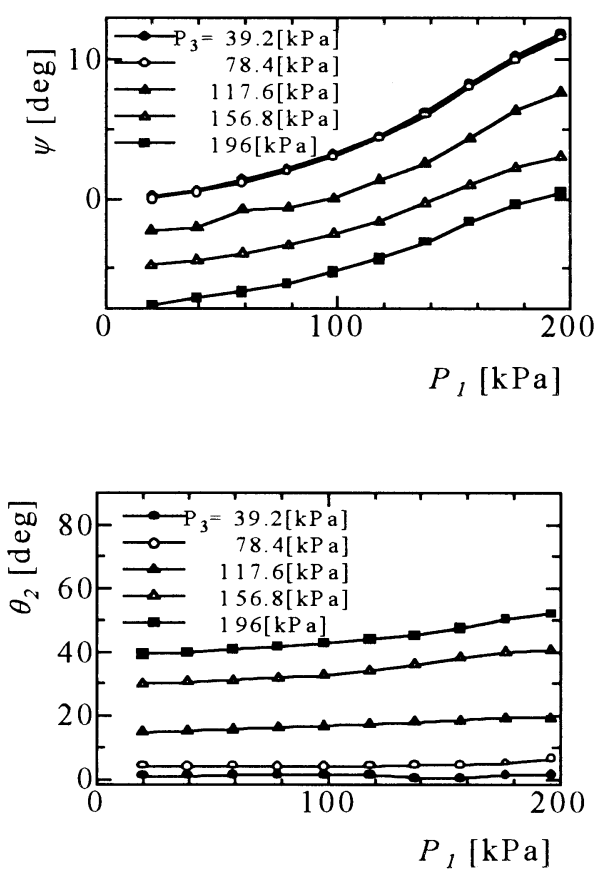

Figure 11: Combination of twist angle $\theta_{2}$ and bending angle $\psi\left(P_{2}=0\right)$

proposed actuator. Although a certain structural interaction among operational axes, the interaction will be decoupled by introducing a feedback control strategy.

This kind of soft actuator can be applied to various driving mechanism. We are now considering its application.

This research has ben partly supported by "Research for the Future" Program of The Japan Society for the Promotion of Science.

\section{REFERENCES}

1.Noritsugu,T, Pneumatic Actuators, Journal of the Robotic Society of Japan, 1997, 15-3, pp.355-359.

2.Noritsugu,T., Human-Friendly Soft Actuator, International Journal of the Japan Society for Precision Engineering, 1997, 31-2, pp.92-96. 